stage IIIC1 (55.7\%). Patients who received adjuvant CMT/RT developed lower vaginal recurrent rates than adjuvant CMT group (1.1\% vs. $9.8 \%$; likelihood ratio $8.05, \mathrm{p}<0.05)$. However pelvic and para-aortic lymph node recurrences, distant recurrences and 2-year overall survival were not different between 2 groups.

Conclusion: Adjuvant chemotherapy followed by radiotherapy offered no benefit for pelvic control or overall survival compared with adjuvant chemotherapy alone for patient with stage III endometrial carcinoma. However, adjuvant chemotherapy followed by radiotherapy reduced vaginal recurrence compared to adjuvant chemotherapy alone.

Poster (E02)

Endometrial Hyperplasia, Endometrial Intra-epithelial Neoplasia, and Endometrial Cancer

https://doi.org/10.3802/jgo.2021.32.S1.E02

\section{Analysis of antitumor activity of dostarlimab by tumor mutational burden (TMB) in patients with endometrial cancer (EC)}

\author{
Audrey Chang,,$^{1, *}$ Ana Oaknin, ${ }^{2}$ Lucy Gilbert, ${ }^{3}$ Anna Tinker, ${ }^{4}$ \\ Jubilee Brown, ${ }^{5}$ Cara Mathews ${ }^{6}$ \\ 'GlaxoSmithKline, Brentford, UK (audrey.x.chang@gsk.com) \\ ${ }^{2}$ Vall d'Hebron Institute of Oncology, Barcelona, Spain \\ ${ }^{3}$ McGill University, Montreal, Canada \\ ${ }^{4} \mathrm{BC}$ Cancer, Vancouver, Canada \\ ${ }^{5}$ Levine Cancer Institute, Charlotte, NC, USA 6 Women \& Infants Hospital of \\ Rhode Island, Providence, RI, USA
}

Objective: GARNET (NCT02715284) is assessing antitumor activity and safety of dostarlimab, an anti-programmed death 1 receptor therapy, in patients with solid tumors. We report on association of antitumor activity and tumor mutational burden (TMB) in 2 endometrial cancer (EC) cohorts.

Methods: A phase 1, multicenter, open-label, single-arm, dose escalation and cohort expansion study. Two expansion cohorts enrolled pts with advanced/recurrent EC (deficient mismatch repair [dMMR; cohort A1] and proficient [MMRp; cohort A2] determined by immunohistochemistry). Patients received dostarlimab $500 \mathrm{mg}$ IV Q3W for 4 cycles, then 1,000 mg IV Q6W until disease progression/discontinuation. Primary endpoint was objective response rate (ORR) by RECIST v1.1. TMB status was an exploratory biomarker determined using the FoundationOne test (TMB-High [TMB-H]: $\geq 10$ mutations/ megabase; TMB-Low [TMB-L]: $<10$ mutations/megabase). Results: Two hundred forty-five patients were included, 103 (TMB-H: 85; TMB-L: 13; TMB missing: 5) in A1 and 142 (TMB-H: 9; TMB-L: 128; TMB missing: 5) in A2. ORRs were $44.7 \%$ (A1) and $13.4 \%$ (A2). In TMB-H pts, ORRs were $44.7 \%$ (A1) and $44.4 \%$
(A2). In TMB-L pts ORRs were $23.1 \%$ (A1) and $11.7 \%$ (A2). Conclusion: TMB-H status was more frequent in $\mathrm{dMMR}$ patients, a known association with these biomarkers, and correlated with higher ORRs in both cohorts. GARNET was not powered to assess antitumor activity by TMB status, and interpretation is limited by the small number of pts in each subgroup. Additional mutational analysis is ongoing.

Funding: GSK 213346; editorial support from Fishawack Health, funded by GSK.

Previously presented at the European Society for Medical Oncology 2021 Annual Meeting, abstract-1458, Oaknin A et al. - Reused with permission.

Poster (E03)

Gynecologic Pathology, Genetics and Epidemiology https://doi.org/10.3802/jgo.2021.32.S1.E03

\section{Analysis of microsatellite instability status and tumor mutational burden in Chinese endometrial cancer patients}

\author{
JiaJia Xu," Ruijia Yang, Chaoran Xia, Zhixiang Yan, \\ Peng Luo, Huiling Wang \\ Topgen Biological Medicine Technology Co., Ltd., Shanghai, China \\ (xujiajia@topgen.com.cn)
}

Objective: Immune checkpoint inhibitors (ICIs), especially pembrolizumab, has yielded encouraging results in recurrent endometrial cancer (EC). Microsatellite instability (MSI) and tumor mutational burden (TMB) are predictive biomarkers for ICIs. In this study, the relationship between the underlying cause(s) of MSI and TMB were investigated in Chinese patients with EC.

Methods: Eighty-six female Chinese patients with EC involved were tested by OncoDrug-Seq ${ }^{\mathrm{TM}}$ 603-gene panel assay through next generation sequencing using Illumina NovaSeq 6000. Fifty patients of ECs with both MSI and TMB available were identified.

Results: There were 11 (12.7\%) individuals who had MSI-H and the rest had MSS. Twenty-one individuals had TMB-H and 112 patients had TMB-L. The median TMB was 12.7. The correlation between TMB and MSI were significant. Patients with MSI-H status had a significantly higher median TMB than patients with MSS status (17.1 vs. 12.7, $\mathrm{p}=0.0006$ ). The correlation between gene mutation of MMR genes ( $M L H 1, P M S 2, M S H 2$, and MSH6) and TMB were further explored 8 of 50 patients carried the four genes mutation was associated with a higher median TMB 77.0 mut/Mb (range=12.0-248 mut/Mb). 4 demonstrated MSH6 mutation (4/8) was associated with a much higher median TMB (96 mut/Mb), while 75\% (3/4) of MSH6 mutations showed MSI-H. 